\title{
Combining survey data with single-dish observations
}

\section{Monica Trasatti*, Gabriele Giovannini}

DiFA, Università di Bologna, Via Ranzani 1, 40127 Bologna, Italy

INAF - ORA Bologna, Via Gobetti 101,40129 Bologna, Italy

E-mail: trasattieira.inaf.it, ggiovanneira.inaf.it

\section{Uli Klein}

AIfA, Universität Bonn, Auf dem Hügel 71, 53121 Bonn, Germany

E-mail: ukleineastro.uni-bonn.de

\section{Annalisa Bonafede}

Hamburger Sternwarte, Universität Hamburg, Gojenbergsweg 112, 21029 Hamburg, Germany

E-mail: annalisa.bonafede@hs.uni-hamburg.de

\section{Federica Govoni, Matteo Murgia}

INAF - ORA Cagliari, Via della Scienza 5, 09047 Selargius (CA), Italy

E-mail: fgovoni@oa-cagliari.inaf.it, matteo@oa-cagliari.inaf.it

Sky surveys represent a fundamental data source for astronomy. Radio surveys, as well as pointed observations, performed with interferometers might be affected by the so-called zero-spacing problem when observing extended sources at relatively high frequencies. This problem cause the most extended features in the source to be filtered out due to the lack of antenna-pairs with small separations. Here we show that interferometric survey data can be successfully combined with pointed observations performed with a single-dish telescope to produce images with high resolution and correct total power information.

The many facets of extragalactic radio surveys: towards new scientific challenges $(*)$

20-23 October 2015

Bologna, Italy

(*) This conference has been organized with the support of the Ministry of Foreign Affairs and International Cooperation, Directorate General for the Country Promotion (Bilateral Grant Agreement ZA14GR02 - Mapping the Universe on the Pathway to SKA)

\footnotetext{
* Speaker.
} 


\section{The combination of interferometric and single-dish data}

Sky surveys are nowadays the largest data generators in astronomy, and this trend is bound to continue with the new generation of instruments. Radio surveys can be performed with both interferometers and single-dish telescopes. The latter ensure the sensitivity to all the emission from an observed field, although at the expense of a good resolution. On the other hand, interferometers provide a higher resolution, but some time at the expense of a good sensitivity to the largest physical spatial scales in the sources. The technical problem of filtering out the most large features in extended sources, known as the zero-spacing problem, might affect also pointed interferometric observations. In general, increasing the observing frequency, interferometers become blind to emissions with physical extension larger than $x(\mathrm{rad})=\lambda / d_{\min }$ due to the lack of antenna pairs with separation smaller than $d_{\min }$ (see Figure 1). Therefore, observing an extended source with embedded discrete point-like sources, an interferometer might be able to recover accurately only the latter. On the other hand, single dishes might be able to recover completely the extended emission but not to resolve the details in the source. The combination of interferometric and single-dish images of the same field allow us to overcome this problem, producing final images with both the high resolution of the former and the short-spacing information provided by the latter. There are different methods to perform the data combination. For a description of the principles and techniques of the combination see, e.g., $[1,2]$. Although some of these technique date back to the '70s/80s, they are far from being routinely used.

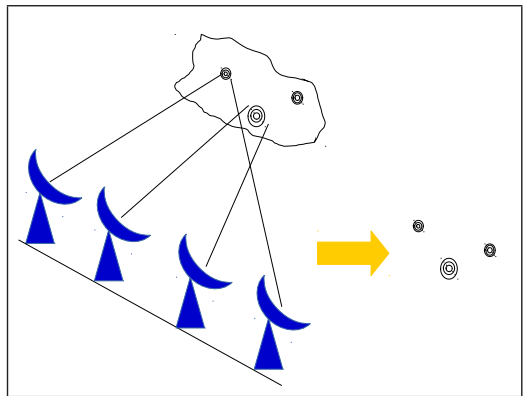

(a) Sketch of interferometric observations.

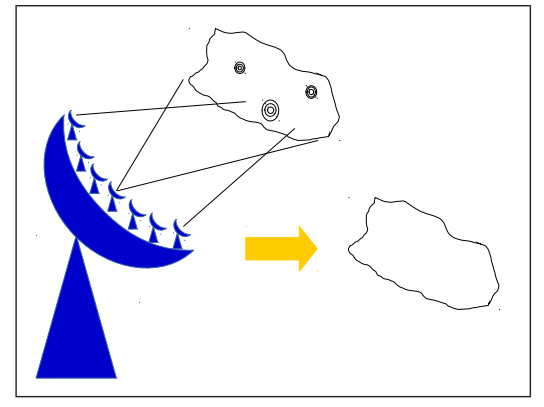

(b) Sketch of single-dish observations.

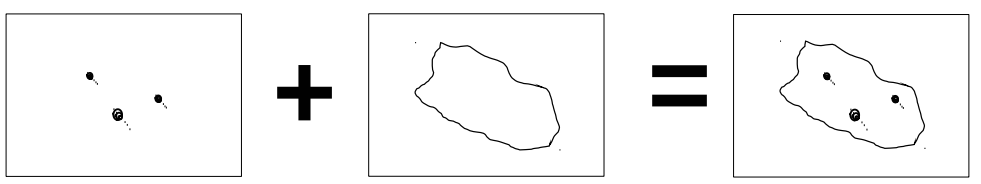

(c) Sketch of the combination technique.

Figure 1: Sketch of interferometric vs single-dish observations.

Here we show the effectiveness of these techniques, and that also data retrieved from surveys can be used for this purpose. We show some of the results we obtained in the study of the extended emission in the Coma cluster. More details on the technique used and on the scientific results are presented in [3]. 


\section{The case of the radio relic in the Coma cluster}

Radio relics are diffuse Mpc synchrotron sources found in the periphery of galaxy clusters (for a review see [4]). They are believed to be produced by (re-)acceleration of particles, by shocks that develop during mergers between clusters. Interferometric observations of diffuse sources, such as radio relics at frequencies around and above $\sim 1 \mathrm{GHz}$, might be especially affected by the zerospacing problem due to their extended nature. However observations at these frequencies yield important constraints on the acceleration mechanism, and have recently showed unusual behavior in some objects $[5,6]$.

Here we show the case of the radio relic in the Coma cluster. The Coma cluster is one of the nearest galaxy cluster, and the first where both a peripheral radio relic and a central radio halo have been observed [7]. Since then, the radio relic has been investigated by several authors in the last decades (e.g. [8], [9]). However high resolution images of the emission are not available at frequencies $>$ $1 \mathrm{GHz}$, due to its large extent and proximity. Figure 2a shows an image of the Coma relic field retrieved from the NRAO VLA Sky Survey (NVSS) [10]. The NVSS is a $1.4 \mathrm{GHz}$ continuum survey performed with the VLA interferometer, covering the entire sky north of $-40^{\circ}$ declination, with a set of 2326 continuum images with $45^{\prime \prime}$ resolution. The image shown is convolved to a resolution of $90^{\prime \prime}$. In the NVSS image most of the extended emission from the relic is filtered out, while the high resolution allow us to resolve the numerous discrete sources present in the field. Figure $2 \mathrm{~b}$ shows an image of the same field obtained from new pointed observations of the Coma cluster performed with the Effelsberg-100m telescope (presented in [3]). In this image the emission from the relic blends with the emission from the galaxies present in the field, due to the low resolution $(9 ! 4 \times 9 ! 4)$. Figure $2 c$ shows the results of the combination in the Fourier domain of the two images $(2 a+2 b)$. The success in recovering the diffuse emission, missed in the NVSS image due to the poor coverage of short baselines, is evident. The flux measured in the relic region in the combined image is $\sim 264 \mathrm{mJy}$ and agrees within $1 \%$ with the flux of the relic as measured from the Effelsberg image. The relic's flux in the NVSS image is only $\sim 75 \mathrm{mJy}$. The combined image has, therefore, the resolution of the NVSS image and the total flux from the Effelsberg image. It is the highest resolution complete image available for this object at this frequency. More details on the combination technique used and on the results obtained are given in [3].

\section{Conclusions}

The study of the extended emission in the periphery of galaxy clusters (relic sources) is important to derive physical properties in low density regions far from the cluster center. The presented results show that using only interferometric observations, necessary to obtain high resolution images, can be dangerous because extended low-brightness regions can be lost. The combination of interferometric and single-dish data is a very powerful tool to study extended radio sources as radio relics at relatively high frequencies. We showed that survey data can be successfully combined with pointed observations to produce images with high resolution and correct total power information.

\section{References}

[1] Stanimirovic S., 1999, PhD Thesis, Arecibo Observatory, NAIC/Cornell University 


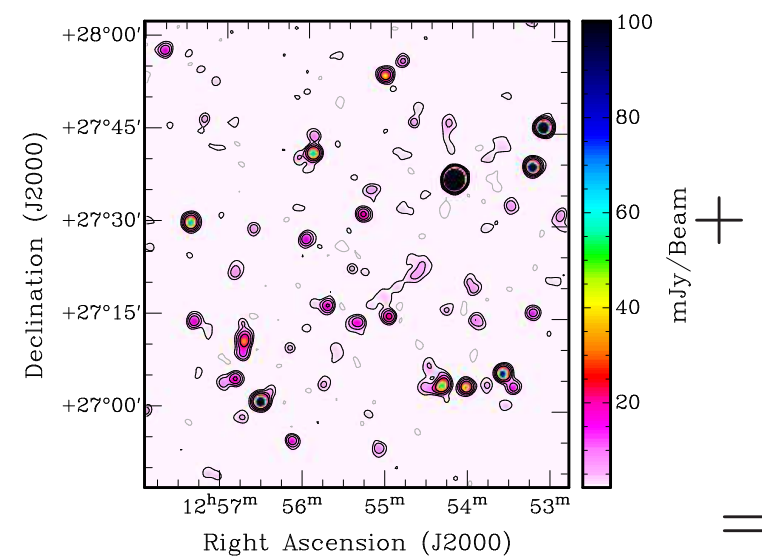

(a) NVSSurvey image.

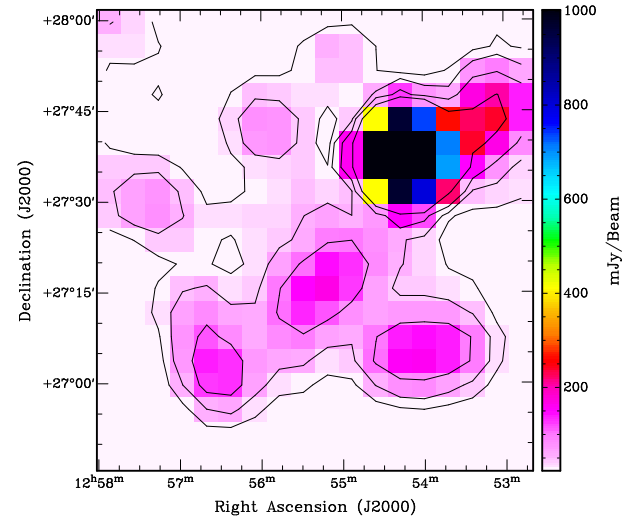

(b) Effelsberg image.

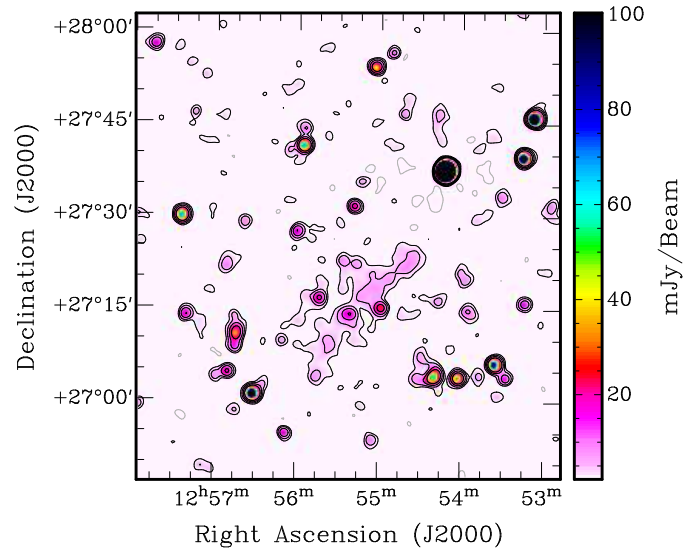

(c) Combined NVSS+Effelsberg image.

Figure 2: $1.4 \mathrm{GHz}$ images of the radio relic in Coma cluster. In panels (a) and (c) contours are drawn at $0.025 \times(-1,1,2,4,8)$ mJy with a resolution of $90^{\prime \prime} \times 90^{\prime \prime}$. In panel (b) contours are drawn at $0.025 \times(1,2,4,8)$ mJy with a resolution of $9.4 \times 9$ '.4.

[2] Trasatti M., 2014, PhD Thesis, Argelander Institut für Astronomie, Universität Bonn

[3] Trasatti M., Klein U., Bonafede A., et al., 2016, submitted

[4] Feretti L., Giovannini G., Govoni F., Murgia M., 2012, A\&ARv, 20, 54

[5] Trasatti M., Akamatsu H., Lovisari L. et al., 2015, A\&A, 575, 45

[6] Stroe A.,Shimwell T., Rumsey C., et al. 2016, MNRAS, 455, 2402

[7] Large M.I., Mathewson D.S., Haslam C.G.T., 1959,Nature, 183, 1663

[8] Giovannini G., Feretti L., Stanghellini C., 1991, A\&A, 252, 528

[9] Brown S., Rudnick L., 2011, MNRAS, 412, 2

[10] Condon J. J., Cotton W. D., Greisen E. W., et al. 1998, AJ, 115, 1693 